\title{
Civilisations
}

Revue internationale d'anthropologie et de sciences

humaines

42-2 | 1993

enQuete d'identité

\section{Identité et patrimoine}

\section{Alain Morel}

\section{OpenEdition}

\section{Journals}

Édition électronique

URL : http://journals.openedition.org/civilisations/2296

DOI : 10.4000/civilisations.2296

ISSN : 2032-0442

\section{Éditeur}

Institut de sociologie de l'Université Libre de Bruxelles

\section{Édition imprimée}

Date de publication : 1 décembre 1993

Pagination : 65-75

ISBN : 2-87263-108-9

ISSN : 0009-8140

\section{Référence électronique}

Alain Morel, «Identité et patrimoine », Civilisations [En ligne], 42-2 | 1993, mis en ligne le 30 décembre 1996, consulté le 19 avril 2019. URL : http://journals.openedition.org/civilisations/2296 ; DOI :

10.4000/civilisations.2296

Ce document a été généré automatiquement le 19 avril 2019

(c) Tous droits réservés 


\title{
Identité et patrimoine
}

\author{
Alain Morel
}

1 Il n'y a pas si longtemps, au nom de la modernité et du progrès, le passé était systématiquement déprécié, disqualifié, et tout ce qui s'y rapportait pouvait être perçu comme de la «vieillerie». Dès les années soixante, nouvelles conditions de vie et désillusions ont entraîné ce que Edgard Morin a appelé «une brèche dans l'adhésion au présent " (Morin, 1967). Conjointement le passé a été reconsidéré et réhabilité, il est devenu l'objet de nostalgie. Vingt ans plus tard, l'intérêt pour le passé a pris la dimension d'un phénomène de masse - en témoigne, par exemple, l'engouement pour les musées dont la fréquentation concurrence celle des stades. Il donne lieu, aujourd'hui, à des investissements de plus en plus importants, notamment sur des fonds publics. Parmi les manifestations multiples de ce phénomène, la mise en valeur des patrimoines culturels, dont on n'a pas fini d'inventer de nouvelles formes. Elle tient à la fois de l'activité militante, qui implique dévouement et croyance. et de l'entreprise culturelle. Qu'est-ce qui motive cette volonté de conserver, et de façon de plus en plus extensive, les traces sous toutes leurs formes, des sociétés et des cultures qui ont précédé celles d'aujourd'hui ? Frénésie de conservation qui conduit les plus prévoyants à vouloir tout sauvegarder, comme s'il fallait se donner les moyens de pouvoir, demain, reconstituer et même reproduire à l'identique le monde d'aujourd'hui, voué lui aussi à une inexorable obsolescence. On sait que l'intérêt pour le passé et la conservation trouvent son origine dans les préoccupations du présent et les éclaire. La construction du patrimoine, sa préservation et sa promotion répondent à d'autres finalités que la seule volonté de perpétuer ce à quoi les présentes générations accordent une valeur certaine et que le temps peut dégrader ou laisser dans l'oubli. Parmi ces finalité, plurielles, il ne sera question ici que de celle qui fait l'objet de ce volume: la construction d'identités collectives.

2 L'investissement social dans la fabrication en tant que telle d'identités personnelles et collectives a pris l'importance qu'on lui connaît aujourd'hui à partir de la fin des années 70. L'intérêt que les chercheurs eux-mêmes ont porté à cette question a suivi la même évolution. Avant 1980, la problématisation en terme d'identification n'intéresse qu'un 
nombre modeste de chercheurs. Les premiers écrits consacrés aux problèmes d'identité remontent à la fin des années 60. Edgar Morin, à partir d'une commune bretonne, procède à un état des lieux d'une culture locale en regard de la modernité dont les normes et les valeurs ont déjà largement pénétré le milieu rural ${ }^{1}$. C'est déjà le temps du retour des migrants installés en ville qui ont changé de point de vue sur la culture de leur aïeux. Celle-ci, après avoir été méprisée, stigmatisée voire réprimée, est redécouverte et revalorisée. Edgar Morin, pour qualifier ce qui se passe à Plozévet, ne parle plus d'acculturation ${ }^{2}$ mais de crise d'identité. Il n'analyse pas la dissonance entre modèles culturels différents en termes de confrontation critique mais en termes de métamorphose: les plodémétiens sont restés, mais ils ne sont plus les mêmes, ils ont changé d'identité : «L'exploitant va se trouver métamorphosé. Ceux qui travaillent la terre ne seront plus les mêmes, oules mêmes ne seront plus paysans " (Morin, 1967 : 131). Il met l'accent sur les altérations du lien social qui s'affaiblit, perd de sa consistance et ne peut plus fonder aussi clairement que par le passé des appartenances reconnues. Il constatait déjà que la recherche de satisfactions individuelles à travers l'investissement dans la maison, la télévision, la voiture - «instruments d'une sur-individualisation» (Morin, $1967: 268$ ) avait pour contrepartie une moindre participation à des collectifs, à des «nous », et un sentiment d'isolement. La conceptualisation en terme d'identité en était à ses débuts. Elle s'est appliquée à des phénomènes de nature différente - confrontation avec la société industrielle, ruptures culturelles, conflits ethniques - qui avaient pour point commun d'avoir des effets déstabilisants sur les individus. Ce sont ainsi retrouvés dans une même rubrique anthropologique, le mal de vivre des classes moyennes, le désespoir des Indiens parqués dans les réserves, le trouble des canadiens français, la détresse des minorités ethniques, et les problèmes de coexistence des groupes malais.

3 Il n'est pas sûr que le phénomène de confrontation de cultures change de nature lorsque, à la différence des situations classiques d'acculturation, milieu d'accueil et milieu d'origine ne sont plus étrangers l'un à l'autre, mais s'inscrivent dans une même culture globale, un même ensemble national. Le passage d'une culture locale, nourrie de traditions à une culture industrielle privilégiant le changement ne pose-t-il pas aussi à ceux qui l'effectuent un problème d'acculturation, même s'il ne s'agit plus d'un rapport d'altérité ethnique? Les mêmes traits se retrouvent d'une analyse à l'autre comme si, partout, les mêmes causes produisaient les mêmes effets. A ce titre, les diverses sociétés, composantes des États modernes, subiraient le même sort que les sociétés du Tiers monde, transformées au contact de la société industrielle occidentale. Toutes auraient perdu quelque chose et de ce fait seraient en état de crise. C. Lévi-Strauss postulait déjà dans les années 40 la généralité du phénomène : «C'est que, s'il existe une infinité de formes de sociabilité, il n'y a qu'une manière de perdre radicalement celle qu'on possède pour en adopter d'autres qui sont imposées du dehors. Et ce processus ne constitue pas une nouvelle forme de sociabilité; c'est une maladie qui est commune à toutes, ou plutôt que toutes sont également susceptibles de contracter » (Lévi-Strauss, 1940-1948: 335). Le processus de déculturation produirait les mêmes effets : "Or ces symptômes sont très peu nombreux : au moment où elles se défont toutes les sociétés convergent, aussi différentes qu'elles aient pu être dans leur état original. Il y a des cultures mélanésiennes, africaines, américaines; la décadence n'a qu'un visage »(idem).

4 Un des symptômes de cette maladie du social n'est-il pas la crise d'identité ? Les problèmes d'identification ont pris l'importance d'une préoccupation générale, concernant de très nombreux individus. Ce serait un des avatars de la société de masse. Les explications données à cette aggravation des problèmes d'identification sont diverses. 
Elles ne considèrent pas le besoin de se distinguer parmi une masse de semblables, fondamentalement différent de celui d'être considéré quand on est au bas de l'échelle.

5 Le besoin - universel - de se différencier ne trouverait plus dans les formes de la société contemporaine de possibilités de s'exprimer. Les diverses cultures locales ont été minorées, dévalorisées, par la diffusion des normes et valeurs de la société de masse. La singularité de leur culture s'est dissoute dans la circulation généralisée des biens, des personnes et des idées. Les savoirs spécifiques propres aux activités locales ne sont plus transmis et les pratiques, qui avaient une valeur emblématique, signes survalorisés à l'aide desquels l'identité s'affiche, tombent en désuétude. De façon encore plus appauvrissante, les activités sociales, insuffisamment différenciées, ne généreraient plus de marqueurs d'identité. La production de la différence doit être développée pour ellemême ; elle devient une activité en soi.

6 Conséquemment, les groupes sociaux sont de plus en plus difficiles à penser et à définir. De plus, il n'est plus possible de les considérer comme des entités homogènes. Comment trouver une identité commune à une collection d'individus qui revendiquent des appartenances multiples ? Comment, en retour, être soi-même perçu comme différent ? Avoir une identité, ce n'est pas seulement avoir la faculté de se distinguer personnellement. C'est aussi appartenir à un groupe distinct qui donne le poids du collectif à la définition de soi-même. Or, ne pas être perçu comme différent c'est ne pas exister socialement. C'est ce que redoute une grande partie de la population, une majorité, qui éprouve de grandes difficultés à se reconnaître et à être reconnue au sein d'une classe moyenne massive et peu différenciée. Elle s'inquiète de l'uniformisation, tant dénoncée. Cette uniformisation, à laquelle participe plus ou moins librement les individus, serait, en même temps, subie et dévalorisante. Comme le dit P. Bourdieu elle se traduit par une perte: "Toute unification qui assimile le différent. enferme le principe dela domination d'une identité sur une autre ... »(Bourdieu, 1980 : 71).

7 A. Touraine voit dans la dissolution des cultures singulières la manifestation d'un phénomène plus radical d'imposition d'une domination: «l'appel à l'identité n'est qu'une forme particulière de la massification et de la déstructuration d'une société deplus en plus soumise au pouvoir absolu de l'Etat» (Touraine, 1984: 175). Il ne s'agit plus tant de se faire reconnaître que de contester la place attribuée dans l'ordre qui s'établit: «l'appel à l'identité apparaît donc d'abord comme un refus de la définition sociale des rôles que doit jouer l'acteur » (Touraine, 1984 : 165).

8 A ces effets de domination et au recul des singularités locales s'oppose la possibilité d'emprunter à diverses cultures locales, globales, internationales, professionnelles, passées, présentes, marchandes, savantes, etc ... , objets, pratiques et signes pour se distinguer et se valoriser? Les possibilités de se différencier semblent même accrues. Les acteurs sociaux ont la faculté, au gré des divers contextes, de donner à leur identité des contenus variables, faisant de celle-ci, comme le dit J.-L. Amsellèà propos de l'ethnonyme, un «signifiant flottant ", susceptible de recevoir des signifiés différents; activité qui consiste tout autant à dissimuler qu'à afficher.

9 Mais cette plus grande liberté, cette plus large autonomie, en ce qu'elles ne répondent pas aux besoins et laissent subsister des problèmes d'identification, révèlent d'autres insatisfactions, d'autres frustrations qui ne se réduisent pas au besoin d'être reconnu dans sa différence. La construction de l'identité à partir de différentes identifications tirées d'une culture médiatisée et des modèles qu'elle propose ou impose semble n'être qu'un artifice, à côté des rapports sociaux réels. Les appartenances se diversifient mais 
elles perdent de leur consistance. Comme le note Ch. Lash (Lash, 1981), l'individu ne peut plus se référer qu'à lui-même, dans un rapport narcissique, que Lash estime névrotique. N'est-il pas nécessaire pour donner corps à son identité, de pouvoir se distinguer du proche, du voisin, de celui que l'on connaît? Pour générer une identification durable et satisfaisante, une culture doit être éprouvée. Elle ne peut seulement être reçue, consommée. La relativisation des normes et des valeurs, soumises au changement social permanent, mine les certitudes et le désir d'adhérer à des modèles. Elle génère le doute sur soi-même. Une identité qui ne résiste pas au temps manque de consistance. Elle doit être ancrée dans la durée, avoir une permanence. L'identité, c'est aussi ce qui perdure par delà les changements.

10 De plus, la diversification des appartenances, la liberté de se définir à sa guise, a pour toile de fond l'individualisme. Il lui correspond un recul des solidarités et une mise à distance des valeurs holistes. Or le besoin de rapports fusionnels est inscrit dans la nature humaine et il faudrait, dit T. Todorov, être "aveugle pour croireque cette appartenance (au groupe) est inutile ou négligeable» (Todorov, 1989). L'identité ne s'éprouve pas que dans l'opposition mais aussi dans la similitude, appréhendée dans des rapports fusionnels, tels, par exemple, que ceux vécus dans les fêtes ou dans l'effervescence des manifestations sportives. A cela s'ajoute l'abandon des grandes idéologies structurantes, politiques et religieuses, qui organisaient le monde et suscitaient solidarités, valeurs, croyances et prises de position, rupture qui ne laisse plus, en point de mire, que le destin personnel. A la place s'insinue un sentiment de vide social. La résurgence des nationalismes participe de cet ensemble de carences.

11 Les préoccupations des maires des villes de banlieue des grandes villes de Provence exprimées en 1985 dans un livre blanc, constituent une illustration de la façon dont la crise culturelle et sociale a pu être perçue et interprétée. Ce livre intitulé «La ville reconquise ", qui résulte d'une réflexion collective d'élus municipaux, est la synthèse de leur analyse des problèmes des villes et quartiers périphériques et des remèdes qu'ils envisagent. : « ... le besoin d'identité, pour être abstrait, n'est pas moins tangible. Lesbesoins d'enracinement dans unesociété locale n'ont pas disparu»(Villes en banlieues: la ville reconquise, 1985 : 58). Ils perçoivent un déficit de lieux de mémoire et de souvenirs collectifs, des espaces publics qui n'ont pas d'âme, des difficultés d'appropriation de territoires qui n'auraient pas de qualités et de limites perceptibles, un manque de discontinuité en quelque sorte. Ils déplorent encore l'absence de vie sociale, l'image floue de la collectivité et le manque d'autonomie, d'indépendance par rapport au centre urbain. Le diagnostic fait état d'un déficit culturel. Ce qui ferait défaut à ces populations - qui ne manquent pas forcément de travail ou de pouvoir d'achat - ce serait un minimum de liens sociaux, de repères, de reconnaissance, qui donne sens au cadre de vie : "Le développement de la vie culturelle constitue, pourles élus des villes de banlieue, un axe privilégié dans leur recherche d'identité: donner une âme à la ville, contribuer à l'émergence d'une communauté et d'une identité propre, assurer à la ville un certain rayonnement, rechercher ses racines, sont autant d'objectifs que les élus se sont fixés à travers une politique culturelle véritable» (idem: 85).

Le phénomène dépasse le cadre des banlieues. Il touche aujourd'hui aussi les diverses sociétés locales qui tendent à n'être plus que des segments de la société globale. Si elles ont gardé des particularités, elles partagent néanmoins la culture commune à l'ensemble de la société, et en ont adopté les comportements et les classements. Aussi ne sont-elles plus des matrices productrices d'un sens endogène susceptible de fonder des identités; 
un sens pour le groupe mais aussi pour les groupes voisins informés des distinctions pertinentes. Les membres de ces sociétés éprouvent, comme ceux des villes, le besoin de fonder leur identité sur quelque chose qu'ils puissent s'approprier et auquel ils puissent donner un sens particulier. Ils s'intéressent de nouveau à leur propre culture et découvrent qu'elle a des vertus différenciatrices, qu'elle peut leur donner une « consistance », pour reprendre un mot de G. Barbichon (Barbichon, 1980: 125), que n'aurait pas le citadin ordinaire.

14 L'autre aspect de ce programme - changer les rapports hiérarchiques entre centre et périphérie en contrebalançant la domination culturelle des classes dominantes associées à la ville-centre par une politique culturelle relativement prestigieuse-illustre bien le problème posé par la crise d'identité : il ne s'agit pas tant de se faire connaître que d'être reconnu positivement; il s'agit de réhabiliter et à leurs propres yeux, l'histoire, la culture, et donc l'identité des habitants de ces espaces périphériques. Et pour ce faire, il faut agir sur les principes de classement (Bourdieu, 1980) et contester à ceux qui le détiennent le pouvoir de définir ce qui a une valeur culturelle, le bien fondé de leur jugement. C'est un rapport de force qui doit mobiliser les représentants de la population. Aussi le terrain de l'identité avec l'hommage aux traditions est-il devenu un passage obligé de la thématique politique.

15 L'interprétation du mal de vivre des banlieues comme un problème d'enracinement, d'appropriation du territoire et d'identification n'est peut être pas sans rapport avec le fait qu'une partie très importante des banlieusards est originaire du milieu rural et qu'une autre partie, elle aussi importante, est issue de l'immigration. Ne s'agit-il pas de la traduction de la nostalgie de populations déracinées pour les sociétés dont elles sont issues, sociétés qui, vues de la mégalopole urbaine, satisferaient justement les manques qu'engendre le mode de vie urbain des classes moyennes. La façon dont les élus et les relais d'opinion sur lesquels ils s'appuient (tels que les représentants d'association par exemple) interprètent la situation, en termes de besoins fondamentaux, besoin de se reconnaître et d'être reconnu, de penser son identité à partir d'un territoire, besoin d'enracinement, besoins ignorés par la logique de développement de la société industrielle, relève des modèles qui ont été construits et de la société traditionnelle supposée assurer une bonne intégration des individus. Modèles, qui, selon J. -L. Amselle, auraient partout la même origine: "Dans les deux cas (tribalisme etrégionalisme), ces mouvements de retour aux sources, d'authenticité, s'enracinent dans la réalité urbaine, ils sont une projection citadine sur une réalité rurale purement imaginaire" (Amselle, 1967: 41). Les auteurs du livre blanc ne font-ils pas que reprendre à leur compte l'interprétation que des populations déracinées et dominées donnent de leur vie sociale, lorsqu'ils proposent comme l'une des deux idées force à retenir : rendre à la ville son identité à travers sa culture et son autonomie par rapport à la ville-centre? Quelle est la motivation ici du terme rendre? Ne s'agit-il pas plutôt de créer, d'inventer de nouvelles formes de solidarité sociale qui soient cohérentes avec la logique dominante de la société?

Revenons sur un point de l'argumentaire des édiles, qui nous conduira à l'autre pôle de cette réflexion: le patrimoine. Les élus font de l'enracinement de la population un élément clé de leur politique. Mais comment «enraciner» des populations si elles n'ont pas pris racine? Que signifie rechercher ses racines lorsqu'on s'adresse à des transplantés? Mais tout d'abord qu'entend-t-on par enracinement?

17 Le phénomène complexe d'enracinement qui implique durée, relations fusionnelles, interconnaissance etc ... est réduit à un de ses aspects, le sentiment d'appartenance à un 
lieu. Un sentiment d'appartenance - point de départ supposé d'un développement social harmonieux - ne peut se développer que s'il est possible d'une manière ou d'une autre de s'approprier le territoire. Appropriation qui ne peut être que symbolique. Or comment s'approprier des espaces réputés sans qualité, sans marque distinctive et pour tout dire sans intérêt? Comment dire son identité à partir de là - le territoire étant une des références les plus immédiates? Il faut s'employer à requalifier, dans tous les sens du terme, ces lieux (dits déshérités). Besoin est d'une culture qui les singularise et les symbolise, et qui permette d'établir une relation entre soi-même et le territoire ? ${ }^{4} \mathrm{La}$ question devient comment dire sa culture? La culture, tirée du local, des lieux, des habitants, réduite à des figures emblématiques plus ou moins élaborées, et presque toujours déproblématisée et figée, est un langage souvent utilisé pour parler de soi. Mais la culture ainsi reconstruite n'aura de vertu qualifiante que s'il lui est conféré une valeur ; il faut qu'elle puisse prendre place à côté d'autres valeurs culturelles reconnues. Dans une société où rien ne dure, où tout se remplace, ce qui justement s'inscrit dans la durée, a une dimension historique, est valorisé. Aussi l'opérateur susceptible d'opérer cette transformation, de conférer des honneurs aux déshérités et un prix à leur culture, est-il le patrimoine.

18 Les caractéristiques du patrimoine le prédispose à une telle utilisation. Il a l'intérêt d'être singulier, d'être attaché à un lieu, et donc de se prêter facilement à une identification emblématique. Il est inscrit dans un plus ou moins lointain passé sinon dans l'Histoire; il confère par association sémantique une valeur à ce qu'il désigne, il distingue; il a l'avantage d'être une notion, comme celle d'identité, aux référents en nombre illimité et il est donc susceptible de s'appliquer à pratiquement n'importe quel objet; enfin dans une société marchande, le patrimoine a aussi l'intérêt d'être un produit culturel consommable par les autochtones et bien sûr par le tourisme. Si la ville n'a pas d'Histoire, il s'est tout de même passé quelque chose, des habitants se sont sûrement distingués, au moins localement. Si la ville n'a pas de vieilles pierres, il est possible de reconstruire de toute pièce un vestige artificiel, comme un vieux moulin, ou de réhabiliter un lieu ordinaire, un lieu de production pour qu'il devienne symbole d'un passé ou d'une culture. S'il n'y a rien à transmettre, il y a encore la mémoire, celle des habitants, qui parée d'une reconnaissance sociale, devient une mémoire collective. Le passé n'est-il pas le moyen le plus immédiat, le plus commode, pour légitimer une identité, pour la faire reconnaitre, surtout quand il est idéalisé ? Il est facile de le transformer dans le sens de ce que l'on veut lui faire dire ou symboliser. Bien souvent est postulée une culture immémoriale, prenant racine dans un lointain passé ; ses transformations successives, qui relativisent sa longévité et son caractère immémorial, fondements de ses qualités valorisantes, sont ignorées et passées sous silence; le recours à des érudits et aux textes anciens, asseoit la légitimité du récit fondateur. Reconstruit, le passé a encore l'intérêt de se prêter à la recherche de consensus. Conflits et divisions n'ont pas été exhumés. L'unanimisme est de rigueur et il favorise l'instauration, a posteriori, de rapports fusionnels: "Bien que l'activité support decette identité professionnelle n'existe plus, ce travail de réappropriation dela mémoire constitue le ciment qui permet d'éviter la désagrégation sociale ... $\|^{5}$.

La culture (au sens anthropologique) des générations précédentes peut avoir, dans cette perspective, une nouvelle utilité ; elle devient, elle aussi, un patrimoine. Un patrimoine, qui a été officiellement reconnu et légitimé, et à qui est attribué, théoriquement, un statut équivalent aux autres formes de patrimoines. Une frontière a été tracée qui place cette culture à une certaine distance. Pas trop loin, pas trop près, à la bonne distance, 
comme le dit Marc Augé, pour pouvoir acquérir le statut d'un patrimoine. Avec ce patrimoine, la relation est ambigüe : il s'agit d'éléments qui ont perdu de leur valeur première, qui n'ont plus le même impact - ils sont devenus inertes - mais qui n'ont pas perdu tout leur sens. "On revient à quelque chose qui est encore soi mais déjà autre altéré 》 (de Certeau, $1974: 141)$.

Cette distance étant prise, pratiques culturelles, objets, rituels, savoirs populaires et techniques, styles régionaux, mémoires et vécus des gens ordinaires, peuvent acquérir le statut d'objets patrimoniaux voués à être étudiés, conservés, exposés. Ils seront requalifiés, et pourront donc faire l'objet d'une réappropriation valorisante. Ils étaient secondairement des marqueurs d'identité, ils avaient aussi d'autres fonctions. Cette distance étant prise, ils n'ont plus que cette fonction: désigner. Ne serait-ce pas ce qui conduit une certaine ethnologie à voir des manifestations d'identité partout?

21 Il est remarquable que la culture locale reconstruite et réinventée soit destinée à un usage externe à la société locale, car une telle culture ne peut plus produire les différenciations internes sur lesquelles se greffaient les identités locales. Il s'agit de donner pour un autre non défini, et non pour la société d'interconnaissance, une image de la collectivité appréciée selon les valeurs de la société dominante, société de plus en plus urbanisée, qui manifeste un engouement pour la vie rurale réenchantée : retour au naturel, au vrai, à la tradition, avec toute la valeur qui s'attache à ce mot aujourd'hui ; ou encore l'image d'une société génératrice d'un autre rapport au monde et à l'autre, une société plus solidaire, plus consensuelle ( on n'avait pas ceci ... oucela.. maison était plus uni»), où le rapport avec les choses et les gens était plus personnel, moins médiatisé, et où, en somme, on savait mieux vivre.

22 La culture locale ainsi reconstruite, le plus souvent avec toutes les transformations que lui ont apporté la mémoire et le changement de perspectives entre la réalité d'hier et celle d'aujourd'hui, est devenue un produit culturel confié à des spécialistes, un produit recyclable dans la société du spectacle et de la consommation. Un produit labellisé patrimoine, propre à participer à la promotion de la collectivité qui en est le légitime détenteur.

L'espace régional semble aujourd'hui l'entité appelée à devenir le groupe d'appartenance de référence. Les grandes villes jouent un rôle déterminant dans cette évolution. Elles misent sur le culturel, dans lequel le patrimoine tient une large place, pour créer une image positive de la région qu'elles animent, et s'approprier l'entité régionale à partir de la culture qui la symbolise. Dans la perspective où les entités culturelles de référence, territorialisées ou non, sont appelées à se renouveler sans cesse, à se défaire et à se refaire, au gré des transformations des unités d'appartenance et d'identification, on est conduit à s'interroger, en conclusion, sur la pérennité des nouvelles formes de patrimoine, construites en références aux entités culturelles d'aujourd'hui. Cela relativise l'intérêt que notre génération porte aux objets culturels à partir desquels est défini et conservé le patrimoine. 


\section{BIBLIOGRAPHIE}

AMSELLE, J. L., 1985, Au coeur de l'ethnie : ethnies, tribalisme et Etat en Afrique, Paris, La découverte.

AUGE, M., 1989, L'autre et le semblable, Paris, Ed. du C.N.R.S.

BARBICHON, G., 1980, «Provinciaux et provinces à Paris, propositions pour l'analyse », in :

Ethnologie française, X, 2.

BOURDIEU, P., 1980, Actes de la recherche en sciences sociales, $\mathrm{n}^{\circ} 35$.

de CERTEAU,M. 1974, La culture aupluriel, Paris, Plon.

LEVI-STRAUSS, C., 1940-1948, L'année sociologique, troisième série.

MORIN, E., 1967, Commune en France, Paris, Fayard.

TODOROV, T., 1989, Nous et les autres, Paris, Le Seuil.

TOURAINE, A., 1984, Le retour de l'acteur, Paris, Fayard.

«Villes en banlieues : la ville reconquise », 1985, in : La documentation française.

\section{NOTES}

1. "Au moment où je fais enquête, une nouvelle transformation a commencé vers 1950-1955. Tout est en mouvement, tout change. La grande transformation française, saisit Plodémet, fait brèche dans la digue qui freinait l'écoulement du temps" (Morin, 1967 : 13).

2. Les phénomènes de confrontation de cultures et les problèmes qu'ils posent aux individus qui ont du adopter, suite à diverses contraintes, des comportements, des manières de penser différents de ceux appris lors de leur première socialisation, étaient alors conceptualisés en terme d'acculturation, chapitre classique de la littérature ethnologique.

3. J.- L. Amselle transpose une analyse de C. Lévi-Strauss développée dans son introduction au livre de M. Mauss, "Sociologie et anthropologie".

4. Pour Marc Augé, culture et identité ne vont pas l'une sans l'autre : "Culture et identité sont deux notions indissociables qui s'appliquent simultanément à la réalité individuelle et à la réalité collective." (Augé, 1989).

5. "Les immigrés comme enjeu des stratégies identitaires locales, Rapport de recherche pour la Mission du patrimoine ethnologique, 1989.

\section{RÉSUMÉS}

How can one interpret the wish to preserve, more and more extensively, the traces, whatever their form, of societies and cultures that have preceded those existing nowadays? What 
signifiance can one give to the unrestrained classification of the culture's common features as heritage? Amongst other finalities, what is taken in consideration here is the social investment for constituting personal and collective identities to which individuals want to give more consistency and consolidation, and even entrenchment. To this end, the past is subject to different operations, the purpose of which are to transfonn it into cultural heritage. Classified as such, objects and social practices acquire the qualifying virtue which is sought : they symbolise and mark out. Local communities appropriate and encourage this extensive classification as cultural heritage.

INDEX

Mots-clés : identité, patrimoine, politiques identitaires, appartenances collectives, culture Keywords : identity, cultural heritage, identity politics, collective belonging

\section{AUTEUR}

\section{ALAIN MOREL}

Mission du Patrimoine Ethnologique, Paris 